\title{
THE CONSTRUCTION OF CERTAIN O-DIMENSIONAL TRANSFORMATION GROUPS
}

\author{
BY \\ R. F. WILLIAMS
}

The purpose of this paper is to demonstrate the power of the techniques of construction introduced in [4] and [6] and hopefully, to encourage new research on the Hilbert-Smith Conjecture. The constructions make use of the functor described in [6] which is useful when a great deal of structure, or much computation is involved, e.g., infinite transformation groups or complicated local cohomology groups.

We give an example in Part I of a 1-dimensional space $X$ (1 for simplicity; similar constructions work for any $n$ ) and a free action of the $p$-adic group $A_{p}$ on $X$ such that

A $. \operatorname{dim} X / A_{p}=m=\operatorname{dim} X+1$ :

B. $H_{c}^{m}(U)=Z_{p}^{\infty}$, for any connected open subset $U$ of $X / A_{p}$.

Property $\mathrm{B}$ is hard to achieve; and this indicates the power of these techniques (e.g., see Lemma 1.3.2).

To see why one is interested in such properties, recall the famous and as yet unsolved

Hilbert-SMITH CONJECTURE. If a compact group $G$ acts freely $\left({ }^{1}\right)$ on a manifold, then $G$ is a Lie group.

To prove this conjecture, it would suffice, [5], [7] or [1], to show that no p-adic group can act freely on a manifold. Thus in the past, researchers have looked for whatever surprising consequences they could find from the assumption that a $p$-adic group $A_{p}$ acts freely on an $n$-manifold $X$. In 1940, P. A. Smith [5] found,

A $. \operatorname{dim} X \mid A_{p} \neq \operatorname{dim} X$.

Later, C. T. Yang [7] found

A $. \operatorname{dim} X^{n} / A_{p}=m=\operatorname{dim} X+2$ (or $\infty$ ); and

B. $H^{m}(U)=Z_{p^{\infty}}, U$ any connected open subset of $X / A_{p}$.

These two properties were also proved in [1] as easy (at least in the free case) consequences of the computation

$$
\begin{aligned}
(*) H^{q}\left(B_{A_{p}}\right) & =Z & & \text { if } q=0 \\
& =Z_{p^{\infty}} & & \text { if } q=2, \\
& =0 & & \text { otherwise }
\end{aligned}
$$

where $B_{A_{p}}$ is the classifying space of $A_{p}$. The proof is as follows: using (*), a wellknown spectral sequence has $Z_{p}$ as a corner term, and out pop $\mathrm{A}_{2}$ and $\mathrm{B}$. Thus,

Received by the editors November 9, 1966.

(1) Here and below we consider only the free case for simplicity. Similar remarks apply to effective transformation groups. 
these two properties are the two most salient consequences of the assumption that $A_{p}$ acts on a manifold.

In [4], Frank Raymond and the author gave an example in which $\mathrm{A}_{2}$ holds. The space of course was not a manifold and B was far from true. The current example represents the author's best attempts to construct an example of a $p$-adic transformation group having these two properties.

Indeed, it is conjectured that $\mathrm{A}_{2}$ and $\mathrm{B}$ cannot occur together for any space $X$, at least if $\operatorname{dim} X \mid A_{p}<\infty$. A weak version of this conjecture is proved in Part II, to wit: the current technique of construction cannot yield an example satisfying $\mathrm{A}_{2}$ and $\mathrm{B}$.

Though very weak, this result is of interest for three reasons: (1) the existence of the example satisfying $\mathrm{A}_{1}$ and $\mathrm{B}$; (2) the "obstruction" is a rather delicate one; and (3) the success of this technique of construction is very nearly necessary for the existence of such an example. That is, this line of reasoning ("if there is an example, then it can be constructed in such-and-such a way") could well lead to a proof of the Hilbert-Smith Conjecture.

Finally we give yet another reason for a renewed attack on this old problem in the form of a compact, 2-dimensional "classifying space" $B_{A_{p}}^{\prime}$, due to E. E. Floyd. The point is, ordinary cohomology does not distinguish between a "real" classifying space and $B_{A_{p}}^{\prime}$, though all the results on this conjecture can be obtained via $H^{*}\left(B_{A_{p}}\right)$ or its isomorph, $H^{*}\left(B_{A_{p}}^{\prime}\right)$. However, complex $K$-theory does distinguish between $B_{A_{p}}$ and $B_{A_{p}}^{\prime}$. Perhaps $K$-theory and some version of the spectral sequence alluded to above could lead to new results on this problem.

0 . Notation and conventions. We will use $S^{n}$ to denote a topological $n$-sphere. $Z$ will be the integers, $Z_{p}=Z / p Z$, and the $p$-adic group $A_{p}$ is the inverse limit of the sequence

$$
Z_{p} \leftarrow Z_{p^{2}} \leftarrow \cdots
$$

in which each map sends a generator into a generator. $Z_{p^{\infty}}$ is the dual of $A_{p}$ and is thus the group of $p^{i}$-roots of unity, $i=1,2, \ldots$.

1 will denote any identity map as well as certain inclusions; if $K$ is an $n$-circuit with boundary, $\partial K$ will denote its boundary. Čech cohomology is used and $H_{c}^{*}$ indicates compact supports. If a group $\pi$ acts on a space $X, X / \pi$ indicates its orbit space. If $\left\{f_{i}\right\}, i=1, \ldots, n$, is a family of periodic maps defined on a subset $A$ of $X$, then we may identify the orbits of $A$ under the group $\pi$ generated by $\left\{f_{i}\right\}, i=1, \ldots, n$, to form a new space $Y$. This device is used throughout.

Finally we will make use of the functor defined in [6]. Let $s^{n}$ be the standard $n$-simplex, thought of as a complex, and hence as closed. A simplex $\sigma$ in a complex $K$ is otherwise always taken as open. $K^{\prime}$ denotes the 1st barycentric subdivision of $K$. The notation differs from that of [6] in that the factors $K, X$ are reversed, which now seems more natural. Thus for an $n$-complex $K$ a space $X$ and a map $q: X \rightarrow s^{n}, K \Delta_{q} X=\{(k, x) \in K \times X: \Phi k=q x\}$ where $\Phi: K \rightarrow s^{n}$ is the simplicial 
map determined on $K^{\prime}$ as follows. A typical vertex of $K^{\prime}$ is the barycenter $b\left(\sigma^{i}\right)$ of some $i$-dimensional simplex $\sigma^{i}$ of $K$. Then $\Phi\left(b\left(\sigma^{i}\right)\right)=v_{i}$, the $i$ th vertex of $s^{n}$, relative to some fixed ordering of the vertices of $s^{n}$. (See [6] for other details.)

\section{An Example Satisfying A 1 AND B.}

\section{Description of the example.}

1.1. First define the sequence $\left\{a_{n}\right\}$ of integers by

$$
a_{1}=1 ; a_{n+1}=a_{n}+n ! \text {. }
$$

Let $\pi_{n}$ be cyclic of order $p^{a_{n}}$. Then $A_{p}$ is the inverse limit of the sequence

$$
\pi_{1} \leftarrow \pi_{2} \leftarrow \pi_{3} \leftarrow \cdots
$$

in which the map $\lambda_{n}: \pi_{n+1} \rightarrow \pi_{n}$ sends a generator, say $g_{n+1}$, of $\pi_{n+1}$ into the generator $g_{n}$ of $\pi_{n}$.

Next, we describe the building blocks:

$$
\begin{aligned}
& X=S^{1} \times[0,1], \\
& B=S^{1} \times 1 \subset X, \\
& q_{0}: X=S^{1} \times[0,1] \rightarrow S^{1} \times[0,1] / B=S^{1} * b=\partial s^{2} * b=s^{2} ; \\
& \phi_{i}: X \rightarrow X \text { the rotation of } S^{1} \text { through } 2 \pi / p^{i} \text { radians, } \\
& r_{i}: X \rightarrow X / \phi_{i}=X .
\end{aligned}
$$

We suppose $X$ triangulated in accordance with [6, S01, p. 322].

1.2. Let $K$ be a triangulated 2-manifold. We successively modify $K$ getting $\left\{K_{n}\right\}$, then $\left\{L_{n}\right\}$. The example will be a limit of the $L_{n}$ 's. As the steps defining $\left\{K_{n}\right\}$ and $\left\{L_{n}\right\}$ are quite complicated, we first briefly describe these for $n=1$ and 2: Let $K$ be a triangulated oriented 2-manifold, say a two-sphere,

$$
\begin{aligned}
& K_{1}=K \Delta_{q_{0}} X, \\
& \left.K_{2}=K \Delta_{q_{1}} X \Delta_{q_{0}} X, \quad \text { (here } q_{1}=q_{0} r_{1}\right), \\
& 1 \Delta r_{1} \Delta q_{0}: K \Delta_{q_{1}} X \Delta_{q_{0}} X \rightarrow K \Delta_{q_{0}} X
\end{aligned}
$$

(see [6, §1]). Then $B_{1}=K \Delta B \subset K_{1}$, and $B_{2}=K \Delta B \Delta X \cup K \Delta X \Delta B \subset K_{2}$, and $K_{i}$ is an orientable manifold with boundary $B_{i}$. Then form $L_{1}$ from $\pi_{1} \times K_{1}$ by identifying the points of $\pi_{1} \times B_{1}$ under $g_{1} \times 1 \Delta \phi_{1} ; \pi_{1}$ still acts on $L_{1}$, with generator induced by $g_{1} \times 1$. Finally $H^{2}\left(L_{1} / \pi_{1}\right)=Z_{p}$.

Form $L_{2}$ by identifying the points of $\pi_{2} \times B_{2} \subset \pi_{2} \times K_{2}$ as follows: the points of $\pi_{2} \times K \Delta B \Delta X$ are identified under the period $p^{2}$ map $g_{2} \times 1 \Delta \phi_{2} \Delta 1$. We identify the points of $\pi_{2} \times K \Delta X \Delta B$ under the group of order $p^{2}$, generated by the two period $p$ maps $g_{2}^{p} \times 1 \Delta \phi_{1} \Delta 1$ and $g_{2}^{p} \times 1 \Delta 1 \Delta \phi_{1} . \pi_{2}$ still acts on $L_{2}$, generated by the map induced by $g_{2} \times 1$. We show below that $H^{2}\left(M_{2} / \pi_{2}\right)=Z_{p^{2}}$ and that the induced homomorphism

$$
\left(\lambda_{1} \times 1 \Delta R_{1} \Delta q_{0}\right)^{*}: H^{2}\left(M_{1} / \pi_{1}\right) \rightarrow H^{2}\left(M_{2} / \pi_{2}\right)
$$

is the usual injection $Z_{p} \rightarrow Z_{p^{2}}$. 
1.3. We return to the general description, and first define:

$$
R_{n}: X \rightarrow X \text { by } R_{n}=r_{n !}
$$

$\left\{b_{i, j}\right\}$, a triangular array of integers, by

$$
\begin{aligned}
b_{11} & =0, \\
b_{n+1, i} & =b_{n, i}+n !, \\
b_{n+1, n+1} & =0 .
\end{aligned}
$$

The maps $q_{i, j}: X \rightarrow s^{2}$ by

$$
q_{i j}=q_{0} r_{b_{i j}} .
$$

(Note $q_{21}=q_{1}$ and $q_{11}=q_{22}=q_{0}$.) We can now define $K_{n}$ :

$$
K_{n}=K \Delta_{q_{n, 1}} X \Delta_{q_{n, 2}} X \Delta \cdots \Delta_{q_{n, n}} X .
$$

From now on we will write $\Delta_{i, j}$ in place of $\Delta_{q_{i j}}$. Note that

$$
1 \Delta R_{n} \Delta \cdots \Delta R_{n} \Delta q_{0}: K_{n+1} \rightarrow K_{n}
$$

is defined and yields

$$
K_{1} \leftarrow K_{2} \leftarrow \cdots
$$

Next let $B_{n}, B_{n, i} \subset K_{n}$ be defined by

$$
\begin{aligned}
& B_{n, i}=K \Delta_{n, 1} X \Delta \cdots X \Delta_{n, 2} B \Delta_{n, i+1} X \Delta \cdots \Delta_{n, n} X ; \\
& U_{i} B_{n, i} .
\end{aligned}
$$

LEMMA 1.3.1. $K_{n}$ is an orientable 2-manifold with boundary $B_{n}$.

Proof. Proceeding by induction, we need only prove:

LEMMA 1.3.2. If $K$ is a triangulated orientable 2-manifold with boundary $\partial K$, then $K \Delta_{i, j} X$ is a triangulated, orientable 2-manifold with boundary $\partial K \Delta_{i, j} X \cup K \Delta_{i, j} B$.

Proof of 1.3.2. There is a map $q_{0}^{\prime}: X \rightarrow s^{2}$ agreeing with $q_{0}$ on $S^{1} \times 0$, mapping $X$ homeomorphically onto $s^{2}-D$, and mapping $B$ onto $\partial D$, where $D$ is a small open disk lying well in the interior of $s^{2}$. Then define $q_{i j}^{\prime}: X \rightarrow s^{2}$ by $q_{i j}^{\prime}=q_{0}^{\prime} r_{b_{i, j}}$. Note that $q_{i j}^{\prime}$ is a local homeomorphism.

Then for any simplex $\sigma$ of $s^{2}, q_{i}^{\prime-1}(\sigma)=q_{i}^{-1}(\sigma)$. It follows (see [6; M1, p. 320]) that both the maps

$$
K \Delta_{q_{i}} X \underset{1 \Delta 1}{\longrightarrow} K \Delta_{q_{i}} X, \quad K \Delta_{q_{i}} X \underset{1 \Delta 1}{\longrightarrow} K \Delta_{q_{i}} X
$$

are defined and are inverses. Hence $K \Delta_{q_{i}} X$ and $K \Delta_{q_{1}} X$ are homeomorphic. Now $K \Delta_{1}\left(s^{2}-D\right) \subset K \Delta_{1} s^{2}$ and this last can be identified with $K$ (actually $K^{\prime}$, the barycentric subdivision of $K\left[6\right.$, p. 320]). Note that $K \Delta_{1}\left(s^{2}-D\right)$ is a manifold with boundary $\partial K \Delta_{1} s^{2} \cup K \Delta_{1} \partial D$. Finally the map $1 \Delta q_{i j}^{\prime}: K \Delta_{q_{i}} X \rightarrow K \Delta_{1}\left(s^{2}-D\right)$ is a local homeomorphism, because $q_{i j}^{\prime}$ is a local homeomorphism. In detail, we need only 
check that $1 \Delta q_{i j}^{\prime}$ is locally $1-1$, by compactness. But if $U$ is open in $X$ and $q_{i j}^{\prime} \mid U$ is $1-1$, then $K \Delta U$ is open in $K \Delta K$ and $1 \Delta q_{i j}^{\prime} \mid K \Delta U$ is $1-1$.

Thus $K \Delta_{q_{i}} X$ is a 2-manifold with boundary $\partial K \Delta_{q_{i}} X \cup K \Delta_{q_{i}} B$, as these are the sets which map onto $\partial K \Delta_{1} s^{2}$ and $K \Delta_{1} \partial D$ under $1 \Delta q_{i j}^{\prime}$. The orientability of $K \Delta_{q_{i}} X$ also follows from the fact that $1 \Delta q_{i j}^{\prime}$ is a local homeomorphism. This last is easily seen by assuming the contrary and looking at an orientation reversing curve in $K \Delta_{q i} X$.

1.4. Note that the map $K_{n+1} \rightarrow K_{n}$ does not send $B_{n+1}$ into $B_{n}$. That is, $B_{n+1, i}$ $\rightarrow B_{n, i}$, for $i \leqq n$, but $B_{n+1, n+1} \rightarrow$ (the barycenters of the two cells of $K_{n}^{\prime}$ ). We call this last set $B_{n, n+1}$, and define $B_{n}^{+}=B_{n} \cup B_{n, n+1}$. We now have a map of pairs $\left(K_{n+1}, B_{n+1}\right) \rightarrow\left(K_{n}, B_{n}^{+}\right)$. These are relative manifolds and our next task is to compute the degree of this map.

Lemma 1.4.1. The map $\left(K_{n+1}, B_{n+1}\right) \rightarrow\left(K_{n}, B_{n}^{+}\right)$is of degree $p^{n \cdot n !}$.

Proof. Proceeding by induction, we need only prove the two lemmas:

LEMMA 1.4.2. If $\left(K_{i}, \partial K_{i}\right), i=1,2$, are triangulated, orientable relative 2-manifolds with $K_{i}-\partial K_{i}$ connected and $f:\left(K_{2}, \partial K_{2}\right) \rightarrow\left(K_{1}, \partial K_{1}\right)$ is a map of degree $m$, then

(a) $\left(f \Delta R_{n}\right):\left(K_{2} \Delta_{n+1, i} X, \partial K_{2} \Delta X \cup K_{2} \Delta B\right) \rightarrow\left(K_{1} \Delta_{n, i} X, \partial K_{1} \Delta X \cup K_{1} \Delta B\right)$ is of degree $m \cdot p^{n !}, i=1, \ldots, n$; and

$$
f \Delta q_{0}:\left(K_{2} \Delta_{n+1, n+1} X, \partial K_{2} \Delta X \cup K_{2} \Delta B\right) \rightarrow\left(K_{1}, \partial K_{1}^{+}\right)
$$

is of degree $m$, where $\partial K_{1}^{+}=\partial K_{1}$ together with all barycenters of the two simplexes of $K_{1}^{\prime}$.

Proof. Just as in the proof of Lemma 1.3.2 we note that the map $\left(f \Delta R_{n}\right)$ is a local homeomorphism with degree locally the degree of $R_{n}: X \rightarrow X$, which is $p^{n !}$. Thus the degree of $\left(f \Delta R_{n}\right)$ is everywhere $\pm p^{n !}$. If the degrees occurred with different signs, they would determine distinct components of the interior of $K_{2}$. But this is connected, as both $K_{2}-\partial K_{2}$ and $X-B$ are connected. This proves (a). The proof of (b) is the same, except that $q_{0}$ has degree 1 , instead of $p^{n !}$.

1.5. Note that $\pi_{i}$ acts on $K_{i}$, so that $A_{p}$ acts on the inverse limit.

One easily sees (e.g., using 3.1.2, below) that the inverse limit of

$$
K_{1} \leftarrow K_{2} \leftarrow K_{3} \leftarrow \cdots \text {, }
$$

is 1-dimensional, as is the limit of

$$
\pi_{1} \times K_{1} \leftarrow \pi_{2} \times K_{2} \leftarrow \cdots .
$$

We next introduce identification among the points of $\pi_{n} \times B_{n} \subset \pi_{n} \times K_{n}$ to form $L_{n}$. This is to be done in such a way that the inverse system

$$
L_{1} \leftarrow L_{2} \leftarrow \cdots
$$

is still defined and still has dimension 1. Clearly $A_{p}$ acts on the second of these limits via $\pi_{i}$ acting on $\pi_{i} \times K_{i}$; this action will induce an action via $\pi_{i}$ on $L_{l}$ and hence an action of $A_{p}$ on the inverse limit. Finally the inverse limit

$$
L_{1} / \pi_{1} \leftarrow L_{2} / \pi_{2} \leftarrow \cdots
$$

is to be 2-dimensional and have the local groups as specified. 
1.6. To specify the identifications among the points of $B_{n}$, we need the sequence $\left\{C^{n}\right\}$ of $n \times n$ matrices $\left\{C_{i j}^{n}\right\}$, defined by

$$
\begin{aligned}
C_{11}^{1} & =1, & & \\
C_{i, j}^{n+1} & =C_{1, j}^{n}+\sum_{k} C_{i, k}^{n}, & i, j & =1, \ldots, n ; \\
C_{n+1, j}^{n+1} & =\sum_{k} C_{1, k}^{n}, & j & =1, \ldots, n+1 ; \\
C_{i, n+1}^{n+1} & =0, & i & =1, \ldots, n .
\end{aligned}
$$

Lemma 1.6.2. $\sum_{j} C_{i j}^{n}=n !, i=1,2, \ldots, n$.

Proof. This is trivially true for $n=1$. By induction, we have, for $i \leqq n$,

$$
\sum_{j} C_{i j}^{n+1}=\sum_{j=1}^{n}\left(C_{i j}^{n}+\sum_{k=1}^{n} C_{1 k}^{n}\right)=n !+n \cdot n !=(n+1) !
$$

For $i=n+1$,

$$
\sum_{j} C_{n+1, j}^{n+1}=\sum_{j=1}^{n+1}\left(\sum_{k} C_{1 k}^{n}\right)=(n+1)(n !)=(n+1) ! .
$$

Next, let $g_{n}(i)$ be the $p^{a_{n}-i}$ power of $g_{n}$. Then

1.6.3. $g_{n}(i)$ has order $p^{i}$.

1.6.4. $\lambda_{n} g_{n+1}(i)=g_{n}(i-n !)$.

Proof. $g_{n+1}(i)=\left(g_{n+1}\right)^{a_{n+1}-i}$ so that $\lambda_{n} g_{n+1}(i)=g_{n+1}^{a_{n+1}-i}=g_{n^{n}}^{a_{n}+n !-i}=g_{n}(i-n !)$.

1.6.5. CoROllary.

$$
\begin{aligned}
\lambda_{n} g_{n+1}\left(C_{i, j}^{n+1}\right) & =g_{n}\left(C_{i, j}^{n}\right), & & i, j \leqq n, \\
& =1 & & \text { otherwise. }
\end{aligned}
$$

This follows directly from 1.6 .4 and the definition of $C_{i, j}^{n+1}$. Writing $\phi(k)$ for $\phi_{k}$ we have

1.6.6. $R_{n} \phi(i)=\phi(i-n !) R_{n}, i \leqq n !$

Proof. $\phi(i)$ is based on a rotation of $2 \pi / p^{i}$ radians; $R_{n}$ multiplies an angle by $p^{n !}$. Thus $R_{n} \phi(i)=\phi(i-n !) R_{n}$.

1.6.7. Corollary.

$$
\begin{aligned}
R_{n} \phi\left(C_{i, j}^{n+1}\right) & =\phi\left(C_{i, j}^{n}\right) R_{n}, & & i, j \leqq n, \\
& =R_{n} & & \text { otherwise. }
\end{aligned}
$$

1.7. Then we let $L_{n}$ be $\pi_{n} \times K_{n}$ with the points of $\pi_{n} \times B_{n}=\pi_{n} \times B_{n, 1} \cup \cdots \cup \pi_{n}$ $\times B_{n, n}$ identified as follows. The points of $\pi_{n} \times B_{n, i}$ are identified under the following maps:

$\begin{array}{ll} & g_{n+1}\left(C_{i, 1}^{n}\right) \times 1 \Delta \phi\left(C_{i, 1}^{n}\right) \Delta 1 \Delta \cdots \Delta 1, \\ & g_{n}\left(C_{i 2}^{n}\right) \times 1 \Delta 1 \Delta \phi\left(C_{i 2}^{n}\right) \Delta 1 \Delta \cdots \Delta 1, \ldots, g_{n}\left(C_{i n}^{n}\right) \times 1 \Delta 1 \Delta \cdots \Delta 1 \Delta \phi\left(C_{i n}^{n}\right),\end{array}$ $i=1,2, \ldots, n$.

LEMMA 1.7.2. The map $\pi_{n+1} \times K_{n+1} \rightarrow \pi_{n} \times K_{n}$ induces a map $L_{n+1} \rightarrow L_{n}$. 
Proof. Look first at the points of $B_{n+1, n+1}$ which are identified to form $L_{n+1, n+1}$. The map $\lambda_{n} \times 1 \Delta R_{n+1} \Delta \cdots \Delta R_{n+1} \Delta q_{0}$ composed with the maps of $(1.7 .1)_{n+1, n+1}$ yield

$$
\begin{aligned}
\lambda_{n} g_{n+1}\left(C_{n+1,1}^{n+1}\right) \times 1 \Delta R_{n} \phi\left(C_{n+1,1}^{n+1}\right) \Delta R_{n} \Delta \cdots \Delta R_{n} \Delta q_{0}, \ldots, \\
\lambda_{n} g_{n+1}\left(C_{n+1, n+1}^{n+1}\right) \times 1 \Delta R_{n+1} \Delta \cdots \Delta R_{n+1} \Delta q_{0} \phi\left(C_{n+1, n+1}^{n+1}\right) .
\end{aligned}
$$

Using Corollaries 1.6.5 and 1.6.7, we have

$$
1 \Delta R_{n} \Delta \cdots \Delta R_{n} \Delta q_{0}, \ldots, 1 \Delta R_{n} \Delta \cdots R_{n} \Delta q_{0} \phi(n !) .
$$

Now since $q_{0}(B)$ is a single point, these equations say that $(1.7 .1)_{n+1, n+1}$-equivalent points are mapped into the same point.

Next look at $\pi_{n+1} \times B_{n+1, i}, i \leqq n$. Then the map $K_{n+1} \rightarrow K_{n}$ composed with the maps of (1.7.1) becomes

$$
\begin{aligned}
\lambda_{n} g_{n+1}\left(C_{i, 1}^{n+1}\right) \times 1 \Delta R_{n} \phi\left(C_{i, 1}^{n+1}\right) \Delta R_{n} \Delta & \cdots \Delta R_{n} \Delta q_{0}, \ldots, \\
& \lambda_{n} g_{n+1}\left(C_{i, n+1}^{n+1}\right) \times 1 \Delta R_{n} \Delta \cdots \Delta R_{n} \Delta q_{0} \phi\left(C_{i, n+1}^{n+1}\right) .
\end{aligned}
$$

Now using Corollaries 1.6.5 and 1.6.7 and the fact that $C_{i, n+1}^{n+1}=0$, we have

$$
g_{n}\left(C_{i, 1}^{n}\right) \Delta R_{n} \phi\left(C_{i, 1}^{n}\right) \Delta R_{n} \Delta \cdots \Delta R_{n} \Delta q_{0}, \ldots, 1 \Delta R_{n} \Delta \cdots \Delta R_{n} \Delta q_{0} .
$$

Thus (1.7.1) $)_{n+1, i}$-equivalent points map into $(1.7 .1)_{n, i}$-equivalent points.

LEMMA 1.8. The action of $\pi_{n}$ on $\pi_{n} \times K_{n}$ induces a free action of $\pi_{n}$ on $L_{n}$.

Proof. On $\pi_{n} \times K_{n}, \pi_{n}$ is generated by $g_{n} \times 1$ which clearly commutes with all the identification maps of (1.7.1), so that this induces an action of $\pi_{n}$ on $M_{n}$. To see that this action is free, note first the obvious criterion:

LEMMA 1.8.1. If $\pi$ and $\rho$ act freely on a space $X$ and commute, then $\pi$ acts freely on $X / \rho$ if and only if the direct product $\pi \times \rho$ acts freely on $X$.

To see that Lemma 1.8.1 applies to Lemma 1.8, note that the ingredients of the maps of $(1.7 .1)_{n, i}$, that is, the $g_{n}\left(C_{i, j}^{n}\right)$ 's and $\phi\left(C_{i, 1}^{n}\right)$ 's all act freely on $B_{n, i}$. Next, a typical element of the group generated by the various maps of $(1.7 .1)_{n, i}$ together with $g_{n} \Delta 1 \Delta \cdots \Delta 1$, is

$$
g_{n}^{k_{0}} g_{n}\left(C_{i 1}^{n}\right)^{k_{1}} \cdots g_{n}\left(C_{i n}^{n}\right)^{k_{n}} \times 1 \Delta \phi\left(C_{i 1}^{n}\right)^{k_{1} \Delta} \cdots \Delta \phi\left(C_{i n}^{n}\right)^{k_{n}}
$$

and for this to be the identity, $\phi\left(C_{i j}^{n}\right)^{k_{j}}=1$, for all $j$. But then $g_{n}\left(C_{i j}^{n}\right)^{k_{j}}=1$ for all $j$, so that finally $g_{n}^{k_{0}}=1$.

1.9. The action of $\pi_{n+1}$ and $\pi_{n}$ on $M_{n+1}$ and $M_{n}$ is equivariant.

Proof. This is just the fact that $\lambda_{n} g_{n+1}=g_{n}$.

We have therefore proved

1.10. $A_{p}$ acts freely on the inverse limit, $L$, of

$$
L_{1} \leftarrow L_{2} \leftarrow L_{3} \leftarrow \cdots \text {. }
$$


2. The 2-dimensional cohomology of $L / A_{p}$. We have shown above that $K_{n}$ is an oriented 2-manifold with boundary $B_{n}$. To study $L_{n} / \pi_{n}$, note that there are two maps $\pi_{n} \times K_{n} \rightarrow L_{n} / \pi_{n}$, forming a commutative diagram:

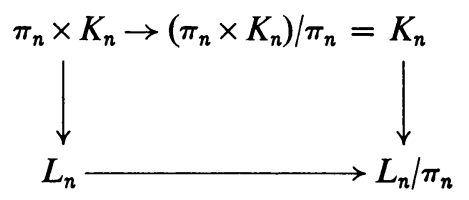

Note that the map $K_{n} \rightarrow L_{n} / \pi_{n}$ is just the quotient map of the equivalences induced on $B_{n, i}$ by those of (1.7.1) on $\pi_{n} \times B_{n i}$, to wit:

$(2.1 .1)_{n, i} \quad 1 \Delta \phi\left(C_{i, 1}^{n}\right) \Delta 1 \Delta \cdots \Delta 1,1 \Delta 1 \Delta \phi\left(C_{i 2}^{n}\right) \Delta 1 \Delta \cdots \Delta 1, \ldots, 1 \Delta 1 \cdots \Delta 1 \Delta \phi\left(C_{i, n}^{n}\right)$.

As these generate a free group action of order $\sum_{j} C_{i j}^{n}=n$ !, we see that $L_{n} / \pi_{n}$ is a relative, oriented manifold with boundary $B_{n}^{\prime}=$ the image of $B_{n} / \pi_{n}$, and with boundary map

$$
L_{n} / \pi_{n} \rightarrow B_{n}^{\prime}
$$

of order $n$ !, i.e.,

LEMMA 2.1.3. $H^{1}\left(B_{n}^{\prime}\right) \rightarrow H^{2}\left(L_{n} / \pi_{n}, B_{n}^{\prime}\right)$ has image $n ! Z$.

Lemma 2.1.4. The map $H^{2}\left(M_{n} / \pi_{n}\right) \rightarrow H^{2}\left(M_{n+1} / \pi_{n+1}\right)$ is the injection

$$
Z_{n !} \rightarrow Z_{(n+1) ! \cdot}
$$

Proof. There is the commutative diagram

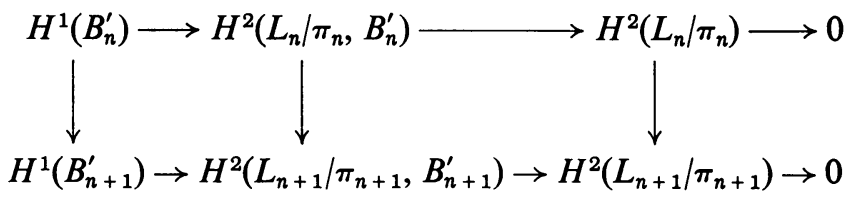

in which the middle map may be replaced by $H^{2}\left(K_{n}, B_{n}\right) \rightarrow H^{2}\left(K_{n+1}, B_{n+1}\right)$ as $\left(K_{n}, B_{n}\right) \rightarrow\left(L_{n} / \pi_{n}, B_{n}^{\prime}\right)$ is a relative homeomorphism. Now by (1.4) and Lemma 2.1.3 the latter part of Lemma 2.1.5 becomes

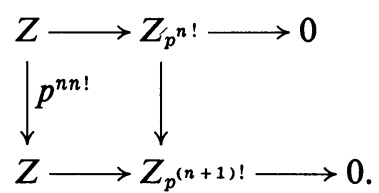

It follows that the last vertical map is an injection, as required. Thus we have proved

THEOREM 2.2. $H^{2}\left(L / A_{p}\right)=Z_{p^{\infty}}$, the p-adic rationals $\bmod 1$.

Actually there is a much stronger result: $\left(H_{c}^{2}\right.$ is Čech cohomology with compact supports):

THEOREM 2.3. $H_{c}^{2}\left(U / A_{p}\right)=Z_{p^{\infty}}$ for any connected invariant open set $U \subset L$. 
Proof. The inverse image of $U$ under the map $A_{p} \times K \rightarrow L$ has the form $A_{p} \times V$, where $V$ is open in $K$, by the invariance of $U$. Now by the definition of the topology for an inverse limit, $U=\left\{x \in L \mid x_{n} \in U_{n}\right\}$, where $n$ is an integer and $U_{n}$ is open in $L_{n}$. Define $U_{n+i}$ by $U_{n+i}=$ inverse image of $U_{n}$ under the map $L_{n+i} \rightarrow L_{n}$. We claim that $U_{n+i}$ is connected. For a separation would lead to a separation of $U$, because the maps $L_{n+1} \rightarrow L_{n}$ are onto, and the $L_{n}$ 's are compact. Similarly one defines $V_{n+i}$ for all $i \geqq 0$ so that $A_{p} \times V_{n+i} \rightarrow U_{n+i}$ under the map $A_{p} \times K_{n+i} \rightarrow L_{n+i}$, and finds that all $V_{n+i}$ are connected.

It follows that $V_{j}$ is an orientable submanifold of $K_{j}$, and that

$$
\left(V_{j+i}, V_{j+1} \cap B_{j+1}\right) \rightarrow\left(V_{j}, V_{j} \cap B_{j}^{+}\right)
$$

is of degree $p^{(j \cdot j !)}$ for $j \geqq n$. This uses the fact that $U_{n+i+1}$ is the complete inverse image of $U_{n+i}$. Now for sufficiently large $i, V_{n+i} \cap B_{n+i} \neq \varnothing$, as $B_{i}$ is $\varepsilon_{i}$-dense in $K_{i}$ where $\varepsilon_{i} \rightarrow 0$ as $i \rightarrow \infty$. But these facts play the role of 1.4 so that one gets analogies of (2.1.2), Lemma 2.1.3, and Lemma 2.1.4 for $U_{j} / \pi_{j}, j \geqq n$. Then Therem 2.3 follows just as Theorem 2.2.

\section{That $L$ is 1 -dimensional.}

3.0. $\operatorname{dim} L \geqq 1$.

Proof. For each $n$, the map $L_{n+1}-B_{n+1}^{\prime} \rightarrow L_{n}-B_{n}^{\prime+}$ is a covering map. Thus the inverse image of a 1-simplex $\sigma_{1}$ under the map $L \rightarrow L_{1}$ is homeomorphic to $\sigma_{1} \times A_{p}$. This is 1-dimensional and thus $\operatorname{dim} L \geqq 1$.

3.1. Thus it suffices to prove $\operatorname{dim} L \leqq 1$. We will do this via a general criterion for an inverse limit to be of dimension $\leqq n$. We also include an analogous criterion for the other inequality.

3.1.1. Definition. Suppose $K, L$ are simplicial complexes and $f: K \rightarrow L$ is a map (not necessarily simplicial). We say $f$ is simplicially of dimension $\leqq n$ provided there is a map $g: K \rightarrow(n$-skeleton of $L)$ such that for all $k \in K, g(k) \in \bar{\sigma}$, where $\sigma$ is the unique open simplex of $L$ containing $f(k)$.

3.1.2. Suppose $X$ is the inverse limit of a sequence

$$
K_{1} \stackrel{f_{1}}{\longleftarrow} K_{2} \stackrel{f_{2}}{\longleftarrow} \ldots
$$

of finite $r$-dimensional complexes, that $f_{i}$ is simplicial relative to a subdivision of $K_{i}$ and that $f_{i}: K_{i+1} \rightarrow K_{i}^{\prime}$ is simplicially of dimension $\leqq n$. Then $\operatorname{dim} X \leqq n$.

Proof. Let $g_{i}: K_{i+1} \rightarrow K_{i}^{\prime}$ be as guaranteed in the definition of simplicially of dimension $\leqq n$ and let $h_{i}: X \rightarrow K_{i}$ be the coordinate map $X \rightarrow K_{i+1}$ followed by $g_{i}$. Then $h_{i}$ maps $X$ into an $n$-complex, for all $i$. Thus it will complete the proof [2, p. 71] to show that $h_{i}$ is an $\varepsilon_{i}$-map, with $\varepsilon_{i} \rightarrow 0$ as $i \rightarrow \infty$.

As this is independent of the metric, we first choose a metric $\rho_{i}$ for $K_{i}$ so that $K_{i}$ has diameter $\leqq 1 / 2^{i}$, and then set $\rho(x, y)=\sum_{i=1}^{\infty} \rho_{i}\left(x_{i}, y_{i}\right)$, for $x, y \in X$. This yields a metric for $X$. Now for each $m, i$ the map $f_{i} f_{i+1} \cdots f_{m}: K_{m+1} \rightarrow K_{i}$ is simplicial relative to the $(m+1-i)$ th-barycentric subdivision of $K_{i}$. Thus if $x, y \in X$, and $x_{m+i}, y_{m+i}$ are in an $r$-simplex $\bar{\sigma}$, then $\rho_{i}\left(x_{i}, y_{i}\right) \leqq(r /(r+1))^{n+1-i} / 2^{i}, i=1,2, \ldots, m$. 
Then $\rho(x, y) \leqq N_{m}=\sum_{j=m+1}^{\infty} 1 / 2^{i}+\sum_{j=1}^{m}(r /(r+1))^{m+1-i} / 2^{i}$. One finds that $N_{m} \rightarrow 0$ as $m \rightarrow \infty$. But this shows that $h_{i}$ is a $\left(1 / 2^{i}+N_{i}\right)$-map and thus completes the proof of 3.1.2.

3.2.1. One says that $f: K \rightarrow L$ is simplicially of dimension $\geqq n$, provided that $f^{*}: H^{n}\left(L, L^{\prime}\right) \rightarrow H^{n}\left(K, f^{-1}\left(L^{\prime}\right)\right)$ is monic for all subcomplexes $L^{\prime} \subset L$.

3.2.2. REMARK. If

$$
K_{1} \longleftarrow f_{1} K_{2} \longleftarrow f_{2} \cdots
$$

is as in 3.1.2 except that $f_{i}$ is simplicially of dimension $\geqq n$, and $\operatorname{dim} K_{1} \geqq n$, then $\operatorname{dim} X \geqq n$.

Proof. One chooses a subcomplex $L$, of $K$, so that $H^{n}\left(K_{1}, L_{1}\right) \neq 0$. Then all the homomorphisms of the system

$$
H^{n}\left(K_{1}, L_{1}\right) \underset{f_{1}^{*}}{\longrightarrow} H^{n}\left(K_{2}, L_{2}\right) \underset{f_{2}^{*}}{\longrightarrow} \cdots
$$

are monic, where $L_{i+1}=f_{i}^{-1}\left(L_{i}\right)$, for all $i$. Hence this limit is nontrivial. But this limit is just $H^{n}\left(X, L_{\infty}\right)$, where $L_{\infty}$ is the inverse limit of $L_{1} \leftarrow L_{2} \leftarrow \cdots$, so that $\operatorname{dim} X \geqq n$.

3.3. We now return to the task of establishing the dimension of our example, and will use the notation of $\S 1$.

3.3.1. LeMmA. The map $L_{n+1} \rightarrow L_{n}^{\prime}$ is simplicially of dimension $\leqq 1$.

Proof. Recall that the map $\pi_{n+1} \times K_{n+1} \rightarrow \pi_{n} \times K_{n}$ is given by

$$
\lambda_{n} \times 1 \Delta R_{n} \Delta \cdots \Delta R_{n} \Delta q_{0}=f_{n} .
$$

We first define $h_{n}$ to agree with $f_{n}$ on $f_{n}^{-1}$ (1-skeleton of $\pi_{n} \times K_{n}^{\prime}$ ). Next, if $\sigma$ is a 2-simplex of $\pi_{n} \times K_{n}^{\prime}$, then $f_{n}^{-1}(\bar{\sigma})$ consists of $p^{(n+1) !}$ copies of $X$. The general fact follows from [6, p. 321] and the specific counting from the definitions of $\lambda_{n}, R_{n}$, (1.1), (1.3).

Let $X_{0}$ be one of these copies of $X$ and let $\eta: X \rightarrow X_{0}$ and $\mu: s^{2} \rightarrow \bar{\sigma}$ be homeomorphisms such that $f_{n} \mid X_{0}=\mu q_{0} \eta^{-1}$. Next let $\rho_{1}, \ldots, \rho_{n+1}$ be the first $n$ maps of $(1.7 .1)_{n+1, n+1}$ and

$$
\nu_{n+1}=g_{n+1}\left(C_{n+1, n+1}^{n+1}\right) \times 1 \Delta 1 \Delta \cdots \Delta 1
$$

Then for each $0 \leqq j_{1}, \ldots, j_{n+1} \leqq p^{n !}-1$,

$$
\rho_{1}^{j_{1}} \cdots \rho_{n}^{j_{n}} \nu_{n+1}^{j_{n}+1} X_{0}=X\left(j_{1}, \ldots, j_{n+1}\right)
$$

is a copy of $X$ which maps onto $\bar{\sigma}$ under $f_{n}$. As $\rho_{i}$ has order $p^{n !}$, by Lemma 1.6.2 and (1.6.3) there are $p^{(n+1) !}$ of these (all different; see Lemma 1.8.1) so that these constitute the total set $f_{n}^{-1}(\bar{\sigma})$.

Now define the map $t(j): X \rightarrow S^{1}=\partial s^{2}$ by

$$
t(j)(\theta, t)=\theta-2 \pi j t / p^{n !}, \quad(\theta, t) \in S^{1} \times[0,1]=X .
$$


Now the $\operatorname{map} f_{n}$ sends

$$
X\left(j_{1}, \ldots, j_{n+1}\right) \rightarrow \bar{\sigma}
$$

according to the formula $\mu q_{0} \eta^{-1} \rho_{1}^{j_{1}} \cdots \nu_{n+1}^{-j_{n+1}}$. We define $h_{n}$ on $X\left(j_{1}, \ldots, j_{n+1}\right)$ to be $\mu t\left(j_{n+1}\right) \eta^{-1} \rho_{1}^{-j_{1}} \nu_{n+1}^{-j_{n+1}}$. As for each $j, t(j)=\dot{q}_{0}$ on $q_{0}^{-1}\left(\partial s^{2}\right)$, which is $S^{1} \times 0 \subset S^{1}$ $\times[0,1]$, this does agree with $h_{n}$ on $f_{n}^{-1}\left(1\right.$-skeleton of $\left.\pi_{n} \times K_{n}^{\prime}\right)$. This then defines $h_{n}$ on all of $\pi_{n+1} \times K_{n+1}$. Having only $\pi_{n} \times K \Delta X \Delta \cdots \Delta X \Delta\left(\partial s^{2}\right)=(1$-skeleton of $\left.\pi_{n} \times K_{n}^{\prime}\right)$ as image.

Note also that $f_{n}(x), h_{n}(x) \in \bar{\sigma}$, where $\sigma$ is the unique simplex containing $f_{n}(x)$, i.e., $h_{n}$ is a "simplicial" approximation to $h_{n}$. Our only hedge is that $h_{n}$ may not be simplicial-which does not harm its value as an approximation.

Finally we wish to show that $h_{n}$ induces a map $L_{n+1} \rightarrow L_{n}$. Thus consider the identifications made on $\pi_{n+1} \times B_{n+1, i}$ in (1.7.1 $)_{n+1, i}$. For $i \leqq n, \pi_{n+1} \times B_{n+1, i} \subset f_{n}^{-1}$ (1-skeleton of $\pi_{n} \times K_{n}^{\prime}$ ) so $h_{n}$ agrees with $f_{n}$ here. For $i=n+1$, there is something to prove; let $\sigma$ be a typical 2-simplex in $\pi_{n} \times K_{n}^{\prime}$, and let the notation $X_{0}, \eta$, $\mu$, et cetera, be as above.

Now $\pi_{n+1} \times B_{n+1, n+1} \cap X\left(j_{1}, \ldots, j_{n+1}\right)=\rho_{1}^{j_{1}} \cdots \rho_{n+1}^{j_{n+1}} \eta(B)=B\left(j_{1}, \ldots, j_{n+1}\right)$. Thus each equivalence class of the relation $(1.7 .1)_{n+1, n+1}$ hits each $B\left(j_{1}, \ldots, j_{n+1}\right)$ in one and only one point. Let $\theta \times 1$ be a point of $B=S^{1} \times 1 \subset X$. Then

$$
\eta(\theta \times 1) \sim \rho_{1}^{j_{1}} \cdots \rho_{n+1}^{j_{n+1}}(\eta(\theta, 1))=\rho_{1}^{j_{1}} \cdots \rho_{n}^{j_{n}} \nu_{n+1}^{j_{n+1}}\left(\eta\left(\theta+2 \pi j_{n+1} / p^{n !}, 1\right)\right)
$$

and these points both go into $\mu(\theta)$. Thus $h_{n}$ induces a map $\bar{h}_{n}: L_{n+1} \rightarrow$ (1-skeleton $L_{n}$ ) and this completes the proof of Lemma 3.3.1.

\section{Why This Construction Cannot Yield $\mathrm{A}_{2}$ AND B}

One can follow the pattern of Part I, $\S 1$ a long way toward the construction of an example satisfying $\mathrm{A}_{2}$ and $\mathrm{B}$. But this process breaks down for a rather subtle reason, and it is this reason that we want to elucidate in this section.

Our method is to present the analogous construction, and show that one of the steps of $\S 1$ cannot be carried out. We will aim at a 2-dimensional example for simplicity even though 3 is the smallest dimension for which a counterexample to the Hilbert-Smith Conjecture could possibly exist [3, p. 249].

4.1. Let $\partial s^{4}=S^{3}=S_{0}^{1} * S_{1}^{1}$ and let $N$ be a small tubular neighborhood of $S_{0}^{1}$. The exact relation between these various structures will be specified below. Note that $S^{3}-N=S^{1} \times D^{2}$, where $D^{2}$ is a 2-disk. We use this in defining

$$
\begin{aligned}
& X=S^{1} \times D^{2} \times[0,1] ; \\
& B=S^{1} \times \partial D^{2} \times[0,1] \cup S^{1} \times D^{2} \times 1 \subset X .
\end{aligned}
$$

$\phi_{i}: X \rightarrow X$ is to be a rotation, relative to $S^{1}$, through an angle of $2 \pi / p^{i}$ radians. Define $q_{0}: X \rightarrow s^{4}$ by

$$
q_{0}:\left(S^{3}-N\right) \times[0,1] \rightarrow S^{3} \times[0,1] \rightarrow S^{3} * b=\partial s^{4} * b=s^{4} .
$$

Here the first map collapses the tubular neighborhood $N$ back down along its fibers to $S^{2}$. The next collapses $S^{3} \times 1$ to a single point $b$, the barycenter of $s^{4}$. Thus $q_{0}$ collapses each orbit of $\phi_{i} \mid B$ to a single point for all $i$. 
$S_{0}^{1}$ is chosen to pass through the barycenters $b_{i}, i=0, \ldots, 4$ of the 3 -simplexes of $s^{4}$, to be orthogonal to the 2-simplexes it hits and to miss the 1-skeleton. Define $r_{i}, q_{i}: X \rightarrow s^{4}$ by

$$
r_{i}: X \rightarrow X / \phi_{i}=X, \quad q_{i}=q_{0} r_{i} .
$$

4.2. One can now repeat the construction just as in Part I, $\S 1$; we will need to consider only $L_{1}$ and $L_{2} . \pi_{1}$ and $\pi_{2}$ are as in Part I, $\S 1$.

Let $K$ be a 4-sphere with a fixed triangulation and let

$$
\begin{aligned}
K_{1} & =K \Delta_{q_{0}} X, \quad B_{1}=K \Delta B \subset K_{1}, \\
K_{2} & =K \Delta_{q_{1}} X \Delta_{q_{0}} X, \\
B_{21} & =K \Delta_{q_{1}} B \Delta_{q_{0}} X, \quad B_{22}=K \Delta_{q_{1}} X \Delta_{q_{0}} B, \\
B_{2} & =B_{21} \cup B_{22} \subset K_{2} .
\end{aligned}
$$

One can show just as in Part I, that $K_{i}$ is an orientable 4-circuit with boundary $B_{i}$, $i=1,2$, and that the map $1 \Delta r \Delta q_{0}:\left(K_{2}, B_{2}\right) \rightarrow\left(K_{1}, B_{1}^{+}\right)$is of degree $p$, where $B_{1}^{+}=B_{1}$ together with a certain 2-dimensional subset, containing the dual 1-skeleton of $K_{1}^{\prime}$.

Form $L_{1}$ from $\pi_{1} \times K_{1}$ by identifying the points of $\pi_{1} \times B_{1}$ under the period $p$ map $g_{1} \times 1 \Delta \phi_{1}$. Then $g_{1} \times 1 \Delta 1$ generates the action of $\pi_{1}$ on $\pi_{1} \times K$ and induces an action on $L_{1}$.

Next consider the identification to be made on $\pi_{2} \times B_{2}$ to yield $L_{2}$ from $\pi_{2} \times K_{2}$. On $\pi_{2} \times B_{21}$ this must be generated by a map $g_{2} \times 1 \Delta \phi_{2} \Delta r$, where $r: X \rightarrow X$ is some map of period $p^{2}$. This is true because the map $\lambda_{1} \times 1 \Delta r_{1} \Delta q_{0}: \pi_{2} \times K_{2} \rightarrow \pi_{1} \times K_{1}$ must send these identifications into those generated by $g_{1} \times 1 \times \phi_{1}$. Now as $q_{0}: X$ $\rightarrow X$ is a homeomorphism away from $B$, it follows that $r \mid X-B$ is the identity. But $X-B$ is dense in $X$ and therefore $\pi_{2} \times K \Delta B \Delta X-B$ is dense in $\pi_{2} \times K \Delta B \Delta X$. Thus $g_{2} \times 1 \Delta \phi_{2} \Delta r=g_{2} \times 1 \Delta \phi_{2} \Delta 1$ throughout $\pi_{2} \times B_{21}$.

So far there has been little choice of maps with which to make identifications. But with $\pi_{2} \times B_{22}$ this changes considerably. Thus we will list all the possible (uniform) choices and show that none works.

4.3. In order that a group action be used for this identification, its orbits must be collapsed under the map to $\pi_{1} \times K \Delta X$. There are three such group actions on $\pi_{2} \times B_{22}=\pi_{2} \times K \Delta X \Delta B$, each of order $p$; they have generators $\gamma_{1}=g_{2}(1) \times 1 \Delta 1 \Delta 1$, $\gamma_{2}=1 \times 1 \Delta \phi_{1} \Delta 1$, and $\gamma_{3}=1 \times 1 \Delta 1 \Delta \phi_{1}$. Now modulo $\pi_{2}, p^{2}$ identifications must be made, in order that property $B$ be satisfied.

Thus the identification group $R$ has as generators

$$
\gamma_{1}^{i_{1}} \gamma_{2}^{i_{2}} \gamma_{3}^{i_{3}}, \quad \gamma_{1}^{j_{1}} \gamma_{2}^{j_{2}} \gamma_{3}^{j_{3}},
$$

where $i_{n}$ and $j_{n}$ are integers $\bmod p$. We may therefore choose another pair of generators of $R$, having the form

$$
\gamma_{2} \gamma_{3}^{i}, \quad \gamma_{1} \gamma_{3}^{j}, \quad i=0,1, \ldots, p-1, \quad j=1,2, \ldots, p-1 .
$$

The case $j=0$ need not be considered as then the group $R$, modulo $\pi_{2}$ would have only order $p$, because $\gamma_{1} \in \pi_{2}$. 
We will distinguish two cases: (1) $i \not \equiv 0 \bmod p$ and (2) $i=0$ but $j \not \equiv 0 \bmod p$. First we must describe some additional structure of the building blocks.

4.4. We next use the fact that the map $L_{2} \rightarrow L_{1}^{\prime}$ must be simplicially of dimension $\leqq 2$, (3.1.1). We will actually work with the map $f: \pi_{2} \times K_{2} \rightarrow L_{1}^{\prime}$, and require that the map $f$ and maps $g$ and $g^{\prime}$ below respect the identifications made in $\pi_{2} \times K_{2}$ to form $L_{2}$.

Thus we assume we have a map $g: \pi_{2} \times K_{2} \rightarrow$ (2-skeleton of $L_{1}^{\prime}$ ) satisfying (3.1.1) and alter $g$ slightly to get a map $g^{\prime}$ which is well behaved on $B_{22}$. Set $g^{\prime}=g$ on $f^{-1}$ (1-skeleton of $L_{1}^{\prime}$ ) as this set does not intersect $B_{22}$. Next, let $\sigma$ be a 4-simplex of $L_{1}^{\prime}$. Then $f^{-1}(\bar{\sigma})$ consists of $p$ copies of $X$ and on each of these $f$ is a copy of the map $q_{0}: X \rightarrow s^{4}$. We will look at one of these copies of $X$ and use this structure with no reference to the copying homomorphism.

Now $S_{0}^{1} \cap\left(2\right.$-skeleton of $\left.s^{4}\right)$ consists of isolated points and if $x \in S_{0}^{1} \cap \sigma^{2}$ is such a point then the fiber $S^{1} \times x^{\prime}$ of the tubular neighborhood $N$ of $S_{0}^{1}$ at this point satisfies $S^{1} \times x^{\prime} \subset \sigma^{2}$. Then $g\left(S^{1} \times x^{\prime}\right) \subset \bar{\sigma}^{2}$ and we may vary $g$ by a homotopy so that $g^{\prime}\left(S^{1} \times x^{\prime}\right)=x \in \sigma^{2}$, maintaining the condition that $g^{\prime}(y) \in \bar{\sigma}$ if $f(y) \in \sigma$. Thus we obtain $g^{\prime}$ which agrees with $f$ on $q_{0}^{-1}$ (2-skeleton $\left.s^{4}\right)$. We can go a bit further and have $g^{\prime}=f$ on a neighborhood of $q_{0}^{-1}$ (2-skeleton of $s^{4}$ ), or what is the same, $g^{\prime}=f$ except near the dual 1-skeleton of $\pi_{2} \times K_{2}$. This enlarges the image of $g^{\prime}$ to $L_{1}^{\prime}-N^{\prime}$, where $N^{\prime}$ is a neighborhood of the dual 1-skeleton of $L_{1}^{\prime}$. This enlargement is harmless, as we may always retract $L_{1}^{\prime}-N^{\prime}$ back to the 2-skeleton of $L_{1}^{\prime}$.

Now return to our copy of $X$. The balance of the $q_{0}\left(S^{1} \times x^{\prime}\right)=x$, where

$$
x^{\prime} \in \partial D^{2} \times[0,1] \cup D^{2} \times 1,
$$

are near the dual 1-skeleton of $s^{4}$. Furthermore they cannot be collapsed to $x$, as this would cover the dual 1-skeleton and it is essential that $g\left(L_{2}\right)$ misses the dual 1 -skeleton. It would therefore appear that $g^{\prime}$ can be so chosen that for such an $x^{\prime}, S^{1} \times x^{\prime}$ maps to $S^{1} \times x$ with degree 1 , where $S^{1} \times x$ is the corresponding fiber of the tubular neighborhood at $x \in s^{4}$. Another rather tedious argument shows that this is indeed the case.

4.5. This supplements (4.1). There is a unique point $y_{i} \in \partial D^{2}$ such that

$$
q_{0}: X=S^{1} \times D^{2} \times[0,1] \rightarrow s^{4}
$$

sends $S^{1} \times y_{i} \times 0$ to $b_{i}, i=0, \ldots, 4$. Then

$$
q_{0}^{-1}(b)=S^{1} \times D^{2} \times 1, \quad q_{0}^{-1}(c)=S^{1} \times y_{i} \times t,
$$

where $c=t b+(1-t) b_{i}, 0 \leqq t<1$. Note that $b$ together with all such $c$ 's constitutes the dual 1-skeleton of $s^{4}$.

In $D^{2}$ we choose a point $y \neq y_{i}, i=0, \ldots, 4$, and five arcs $\alpha_{i}, i=0, \ldots, 4$, where $\alpha_{i}$ has end points $y$ and $y_{i}$, and $\alpha_{i} \cap \alpha_{j}=\varnothing, i \neq j$. Let $W=\bigcup_{i=0}^{4}\left(S^{1} \times y_{i} \times[0,1] \cup S^{1}\right.$ $\left.\times \alpha_{i} \times 1\right)$. Then $q_{0}(W)=\left(\right.$ dual 1-skeleton of $\left.s^{4}\right)=s k_{1}$ and $W$ is homeomorphic to $S^{1} \times s k_{1}$. Also the projection $q_{0}^{\prime}: S^{1} \times s k_{1} \rightarrow s k_{1}$ differs only a little from $q_{0}$. In 
particular, $q_{0}(w)$ and $q_{0}^{\prime}(w)$ lie in the same open simplex of $s^{4}$ for each $w \in W$. Finally, note that $\phi_{i}$ leaves $W$ invariant and that $\phi_{i}$ on $W=S^{1} \times s k_{1}$ is just a rotation on the first factor.

4.6. Case $1, i \neq 0 \bmod p$. We choose an $\operatorname{arc} \beta$ in the space $K \Delta q_{1} X \Delta_{1} s k_{1}$ with end points of the form $x_{0}$ and $1 \Delta \phi_{1} \Delta 1\left(x_{0}\right)$. $\left(1 \Delta \phi_{1} \Delta 1\right)$ does act on $K \Delta X \Delta s k_{1}$. Let $\beta^{+}=\beta$ together with its iterates under $1 \Delta \phi_{1} \Delta 1$. Then $\beta^{+}$is a loop; we will assume that $\beta^{+}$ is a topological 1-sphere, which it is if $\beta$ is carefully chosen. Now

$$
1 \Delta 1 \Delta q_{0}: K \Delta_{q_{1}} X \Delta_{1} w \rightarrow K \Delta_{q_{1}} X \Delta_{1} s k_{1}
$$

and that part which maps onto $\beta^{+}$is $S^{1} \times \beta^{+}$with $1 \Delta 1 \Delta q_{0} \mid S^{1} \times \beta^{+}$being essentially the projection.

Now $S^{1} \times \beta^{+} \subset K \Delta X \Delta W \subset K \Delta X \Delta B \subset B_{22}$, and $\gamma_{2} \gamma_{3}^{i}$ leaves $S^{1} \times \beta^{+}$invariant. It is the specific way in which $\gamma_{2} \gamma_{3}^{i}$ acts on $S^{1} \times \beta^{+}$that makes it impossible to carry out this construction. To wit, $\gamma_{3}^{i}$ effects a rotation of $S^{1}$ through an angle of $2 \pi i / p$ radians and $\gamma_{2}$ a rotation of $\beta^{+}$through an "angle" of $2 \pi / p$.

Now $g^{\prime}$ maps the torus $S^{1} \times \beta^{+}$to another $S^{1} \times \bar{\beta}$ in $L_{1}^{\prime}$ and this map is of type $(1, p)$, i.e., of degree 1 on $S^{1}$ (proved in 4.4) and of degree $p$ on $\beta^{+}$. Next choose coordinates $(\theta, \phi)$ for $S^{1} \times \beta^{+}, \theta, \phi$ in the reals mod 1 , and let $S^{\prime}$ be the set of all $(\theta, i \theta)$ in $S^{1} \times \beta^{+}$. Then $S^{\prime}$ is a 1 -sphere wrapping once around the $S^{1}$ factor and $i$ times around the $\beta^{+}$factor. Then $\gamma_{2} \gamma_{3}^{i}(\theta, i \theta)=(\theta+1 / p, i \theta+i / p)$ so that $S^{\prime}$ is invariant under $\gamma_{2} \gamma_{3}^{i}$. Now as the points of $S^{\prime}$ are identified (in forming $L^{2}$ ) we see that $g^{\prime}$ takes $S^{\prime}$ to $S^{1} \times \bar{\beta}$ by a map of type $(p k, p)$. But by the above $g^{\prime} \mid S^{\prime}$ is of type $(i, p)$. This contradicts the fact that $i \neq 0 \bmod p$.

4.7. It remains only to consider case 2 , i.e., $i \equiv 0 \bmod p$ but $j \not \equiv 0 \bmod p$. The argument in this case is much the same, except that $\beta^{+}$must be defined differently. We choose an $\operatorname{arc} \beta$ in $K \Delta_{q_{1}} X \Delta_{1} s k_{1}$ with endpoints of the form $x_{0}$ and $\left(1 \Delta \phi_{1} \Delta 1\right) x_{0}$. Now let $\beta^{+}$be $\beta$ together with its iterates in the space $\pi_{2} \times K \Delta_{q_{1}} X \Delta s k_{1}$ under the map $g_{2}(1) \times 1 \Delta \phi_{1} \Delta$. (See 4.3.) Then $\gamma_{1} \gamma_{2} \gamma_{3}^{j}$ leaves $S^{1} \times \beta^{+}$invariant. Proceeding as in (4.6) we find a contradiction to the fact that $j \neq 00 \bmod p$. This completes the proof.

\section{Classification Spaces for $A_{p}$}

5. A compact, 2-dimensional classifying space. The following construction is due to E. E. Floyd. Let $p$ be a fixed prime and let $n_{i}=p^{2^{i}}, i=0,1, \ldots$ We will construct regular, 2-dimensional $C W$-complexes $K_{i}$, free actions of $Z_{n_{i}}$ on $K_{i}$ and maps $f_{i}: K_{i+1} \rightarrow K_{i}$, equivariant with respect to the usual epimorphism $Z_{n_{i+1}} \rightarrow Z_{n_{i}}$. Let $K_{i}=Z_{n_{i}} * S_{i}$, where $*$ denotes the join and $S_{i}$ is a 1 -sphere. $Z_{n_{i}}$ acts on $K_{i}$ by translation on $Z_{n_{i}}$ and by rotation through an angle of $2 \pi / n_{i}$ on the second factor. Thus $K_{i}$ is $S_{i}$ together with $n_{i}$ oriented 2-cells, $\left\{x e_{i}: x \in Z_{n_{i}}\right\}$ with the boundary formula $\partial\left(x e_{i}\right)=S_{i}$, for all $x \in Z_{n_{i}}$ and $i=0,1,2, \ldots$.

The map $f_{i}: K_{i+1} \rightarrow K_{i}$ sends the 1-skeleton $S_{i+1}, n_{i}$ times around $S_{i}$, and is thus equivariant on the 1-skeleton. To define $f_{i}$ on the 2-cells consider $e_{i+1}=1 e_{i+1}$, let 
$T$ generate $Z_{n_{i+1}}$ and $t$ generate $Z_{n_{i}}$. We think of $e_{i+1}$ as $\left[0,2 n_{i}-1\right] \times S^{1} / 0 \times S^{1}$. First collapse $(2 k) \times S^{1}$ to points, $k=1,2, \ldots, n_{i}-1$. This yields a "string" of $n_{i}-12$-spheres $S_{k}^{2}=[2 k-1,2 k] \times S^{1}$, with ends collapsed, and one disk

$$
\left[2 n_{i}-2,2 n_{i}-1\right] \times S^{1} /\left(2 n_{i}-2\right) \times S^{1} \text {. }
$$

Then $f_{i}$ sends this disk to $n_{i} t^{n_{i}-1} e_{i}$, extending $f_{i}$ already defined on its boundary, $\left(2 n_{i}-1\right) \times S^{1}$. We are now fairly free to map the spheres $S_{k}^{2}$ where we will, and send $S_{k}^{2}$ to the oriented sphere $t^{k-1} e_{i}-t^{k} e_{i}$ with degree $k$. Thus, on the chain level,

$$
e_{i+1} \rightarrow \sum_{k} k\left(t^{k-1}-t^{k}\right) e_{i}+n_{i} t^{n_{i}-1} e_{i}
$$

Thus $f_{i \#}\left(e_{i}\right)=\left(1+t+\cdots+t^{n_{i}-1}\right) e_{i}=\sigma e_{i}$ where $\sigma=1+t+\cdots+t^{n_{i}-1}$, as usual. Define $f_{i}$ on the remaining 2-cells $\left\{x e_{i+1}: x \neq 1\right\}$ by equivariance:

$$
f_{i}\left(x e_{i+1}\right)=x f_{i}\left(e_{i+1}\right) \text {. }
$$

Then on the chain level $f_{i \#}\left(x e_{i+1}\right)=x \sigma e_{i}=\sigma e_{i}$. Now $K_{i+1}$ has the homotopy type of a wedge of $n_{i+1}-12$-spheres, which we take to be

$$
\Sigma_{j}^{2}=t^{j}(1-t) e_{i+1}, \quad j=0,1, \ldots, n_{i+1}-2 .
$$

Then $f_{i}$ sends $\Sigma_{j}^{2}$ to $\sigma t^{j}(1-t) e_{i}$ and is thus of degree zero on each of the $n_{i}-1$ 2-spheres which make up $K_{i}$, again up to homotopy type. Thus the map $f_{i}^{*}: h\left(K_{i}\right)$ $\rightarrow h\left(K_{i+1}\right)$ is trivial in any generalized cohomology theory. Let $E^{\prime}$ be the inverse limit of $K_{0} \leftarrow K_{1} \leftarrow \cdots$. Then $A_{p}$ acts freely on $E^{\prime}$ and we have proved the

5.1. Proposition (Floyd). There is a 2-dimensional compact space $E^{\prime}$ and a free action of $A_{p}$ on $E^{\prime}$ where $E^{\prime}$ is a cyclic in any cohomology theory, defined on the category of finite $C W$-complexes and extended in the Cech manner to compact pairs.

5.2. Corollary (SEe also [1]).

$$
\begin{aligned}
H^{q}\left(B_{A_{p}}\right) & =Z, & & q=0, \\
& =Z_{p^{\infty}}, & & q=2, \\
& =0 & & \text { otherwise. }
\end{aligned}
$$

Proof. By the usual obstruction theory arguments, $H^{*}\left(B_{A_{p}}\right)=H^{*}\left(B_{A_{p}}^{\prime}\right)$ where $B_{A_{p}}^{\prime}=E^{\prime} / A_{p}$. But $E^{\prime} / A_{p}$ is the inverse limit

$$
P_{p} \leftarrow P_{p^{2}} \leftarrow \cdots
$$

in which each $P_{k}$ is a 1 -sphere with a 2 -cell attached by a map of degree $k$. The map $P_{n_{i+1}} \rightarrow P_{n_{i}}$ sends the 2-cell $\left[e_{i+1}\right]$ to $n_{i}\left[e_{i}\right]$. Hence, in the integral cohomology $H^{2}\left(E^{\prime} \mid A_{p}\right)$ is the direct limit of

$$
Z_{p} \rightarrow Z_{n_{1}} \rightarrow \cdots
$$

in which the maps are the usual injections. This completes the proof of 5.2.

6. The complex $K$-theory of $B_{A_{p}}$ and $B_{A_{p}}^{\prime}$.

6.1. $\widetilde{K}^{0}\left(B_{A_{p}}^{\prime}\right)=Z_{p^{\infty}}$, with trivial products. 
Proof. We let $[X, Y]$ denote the set of all homotopy classes of base point preserving maps of $X$ into $Y$. Then, using the spaces $P_{m}$, defined in (5)

$$
\left[P_{m}, B U\right] \approx\left[P_{m}, B U(1)\right]=\left[P_{m}, C P^{\infty}\right] \approx\left[P_{m}, C P^{1}\right]=\left[P_{m}, S^{2}\right]
$$

inasmuch as $P_{m}$ is 2-dimensional. Finally, by the Hopf classification theorem $\left[P_{m}, S^{2}\right] \approx Z_{m}$, and the map $P_{n_{i+1}} \rightarrow P_{n_{i}}$ induces the injection $Z_{n_{i}} \rightarrow Z_{n_{i+1}}$. This completes the proof of 6.1 .

The "usual" classifying space, $B_{A_{p}}$ (e.g., see [1]) can be achieved as a double limit in 2 ways:

(1) $\mathrm{I} \mathrm{L}_{i} \mathrm{D} \mathrm{L} \mathrm{L}_{j}\left(S^{2 i+1} / Z_{p^{j}}\right)$,

(2) $\mathrm{D} \mathrm{L} \mathrm{L}_{j} \mathrm{~L} \mathrm{~L}_{i}\left(S^{2 i+1} / Z_{p^{j}}\right)$.

Here I $\mathrm{L}$ means inverse limit and $\mathrm{D} L$, direct limit. The action of $Z_{p^{j}}$ on $S^{2 i+1}$ is that obtained by considering $S^{2 i+1}$ as the unit sphere in complex $(i+1)$-space. These two ways give two ways of "calculating" $K\left(B_{A_{p}}\right)$. The first yields $\widetilde{K}^{0}\left(B_{A_{p}}\right)$ $\approx Z_{p^{\infty}}$, just as above. This can be seen in various ways, e.g., an easy spectral sequence argument, using the data found in [1].

The second yields

$$
\tilde{K}^{0}\left(B_{A_{p}}\right) \approx \tilde{R}\left(Z_{p^{\infty}}\right) \otimes A_{p},
$$

in which $\widetilde{R}$ is the reduced representation ring. This is a deeper computation, and is due to Don Anderson. Note this ring is torsion-free; more striking, it has arbitrarily long nontrivial products, $x_{1} \otimes \cdots \otimes x_{n}$. This in particular means $B_{A_{p}}$ is infinite dimensional.

Perhaps this last fact can be used to show that if $A_{p}$ acts on a manifold $M$, then $M / A_{p}$ is infinite dimensional. So far, all the author's attempts have failed. Indeed, there is the easy

6.2. RemarK. If $A_{p}$ acts freely on an $n$-manifold $M$, then the dimension of $M / A_{p}$ is $n+2$, relative to any cohomology theory which satisfies the Vietoris mapping theorem.

Proof. There is the diagram

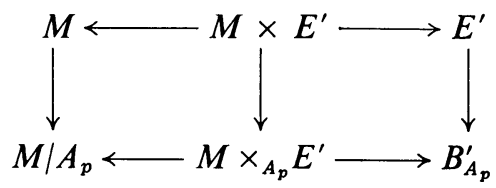

in which $M \times_{A_{p}} E^{\prime}$ is $(n+2)$-dimensional over integral cohomology, and at most $(n+2)$-dimensional because of the map $M \times_{A_{p}} E^{\prime} \rightarrow B_{A_{p}}^{\prime}$, see [2, p. 91]; hence $\operatorname{dim} M \times_{A_{p}} E^{\prime}=n+2$. But the map $M \times_{A_{p}} E^{\prime} \rightarrow M / A_{p}$ is a Vietoris map.

\section{BIBLIOGRAPHY}

1. Glen E. Bredon, Frank Raymond and R. F. Williams, p-adic transformation groups, Trans. Amer. Math. Soc. 99 (1961), 488-498.

2. W. Hurewicz and H. Wallman, Dimension theory, Princeton Univ. Press, Princeton, N. J., 1948. 
3. D. Montgomery and L. Zippin, Topological transformation groups, Interscience, New York, 1955.

4. Frank Raymond and R. F. Williams, Examples of p-adic transformation groups, Ann. of Math. 78 (1963), 92-106.

5. P. A. Smith, Periodic and nearly periodic transformations, Lectures in Topology, Univ. of Michigan Press, Ann Arbor, 1941.

6. R. F. Williams, $A$ useful functor and three famous examples in topology, Trans. Amer. Math. Soc. 106 (1963), 319-329.

7. C. T. Yang, p-adic transformation groups, Michigan Math. J. 7 (1960), 201-218.

NORTHWESTERN UNIVERSITY,

Evanston, Illinois 